\title{
Georg Simmel, Stefan George UND DER ERSTE WELTKRIEG
}

Gerd Hammer

(Universidade de Lisboa)

\begin{abstract}
Von allen schlimmen Folgen aber, die der letzte mit Frankreich geführte Krieg hinter sich drein zieht, ist vielleicht die schlimmste ein weitverbreiteter, ja allgemeiner Irrtum: der Irrtum der öffentlichen Meinung und aller öffentlich Meinenden, daß auch die deutsche Kultur in jenem Kampfe gesiegt habe und deshalb jetzt mit den Kränzen geschmückt werden müsse, die so außerordentlichen Begebnissen und Erfolgen gemäß seien. Dieser Wahn ist höchst verderblich: nicht etwa, weil er ein Wahn ist-denn es gibt die heilsamsten und segensreichsten Irrtümer - sondern weil er im Stande ist, unseren Sieg in eine völlige Niederlage zu verwandeln: in die Niederlage, ja Exstirpation des deutschen Geistes zu Gunsten des deutschen Reiches. (Nietzsche 1980, 137)
\end{abstract}

Am 11. November des Jahres 1918 vor 100 Jahren endete der Erste Weltkrieg. Und die Erinnerungen und Feierlichkeiten dieses Ereignisses fielen weit spärlicher aus als die zu seinem Anfang vier Jahre zuvor. Am 26. September 2018 jährte sich auch der Tod Georg Simmels zum einhundertsten Male. Anlass, noch einmal der Frage nachzugehen, was diesen bedeutenden Soziologen und Philosophen dazu bewegt hat, den Ersten Weltkrieg anfangs zu begrüßen. Als Ergänzung dazu soll der Frage nach der Haltung zum Krieg auch bei Stefan George nachgegangen werden, der diesen Krieg vorhersah, nicht gänzlich abgelehnt, sich daran aber auch nicht beteiligt hat.

1 Departamento de Estudos Germanísticos da FLUL / CEC.

GerdHammer@letras.ulisboa.pt

Philosophica, 53, Lisboa, 2018, pp. 79-89. 
Das Jahr 2014 führte zu einer immensen Produktion historischer Arbeiten, die sich dem Ersten Weltkrieg, dieser Urkatastrophe des 20. Jahrhunderts, als die ihn der amerikanische Historiker George F. Kennan 1979 bezeichnete, widmen. Eine der bekanntesten Studien dazu stammt von Christopher Clark: The Sleepwalkers. How Europe went to war in 1914. Das umfangreiche Buch mit seinem wohl etwas unglücklich gewählten Titel sieht die Kriegsschuld nicht mehr vorrangig im Militarismus des deutschen Kaiserreichs sondern als eine Verquickung unglücklicher Umstände in angespannter weltpolitischer Lage, ein sich gegenseitiges Hochschaukeln. Damit unterscheidet sich das Buch nicht von den meisten der neueren Publikationen, die sich als eine Relativierung deutscher Kriegsschuld lesen lassen, nicht mehr als deutschen „Griff nach der Weltmacht“, so der Titel des berühmten, 1961 erschienenen Buchs von Fritz Fischer. In den Sleepwalkers sucht man die Namen Georg Simmel, Stefan George oder etwa auch Thomas Mann vergeblich. Dies ist bedauerlich, denn hier lassen sich Varianten konservativer und nationalistischer Kriegsbegeisterung und Kriegsverweigerung finden, die weniger mit Pazifismus oder Imperialismus, aber viel mit deutschem Geist und der Ästhetik der Jahrhundertwende zu tun haben.

Der Ausbruch des Ersten Weltkriegs wurde in Deutschland vor allem von Bürgern, Künstlern, Schriftstellern und Philosphen enthusiastisch begrüßt, Arbeiter und die ländliche Bevölkerung sahen den Krieg wesentlich skeptischer. Paul Natorp, einer der Gründer des Marburger Neukantianismus, wollte „das Morgenwehen eines neuen Tages nicht bloß für Deutschland, sondern für die Menschheit" (Natorp 1918, 176) verspürt haben, der Philosoph und Literaturnobelpreisträger Rudolf Eucken, einer der bekanntesten Vertreter des Neufichteanismus, sah die Chance für den deutschen Menschen gekommen. Auf über 400 Seiten präsentierte Max Scheler seinen Nationalismus unter dem Titel „Genius des Krieges und der deutsche Krieg“. Kriegsbegeistert, aber auch zweifelnd meldet sich Max Weber sofort am 2. August 1914 freiwillig zum Kriegsdienst. Wiederholt schreibt er, wie groß und wunderbar dieser Krieg doch sei.

Nur wenige Stimmen wagen es, sich für den Frieden einzusetzen, eine kleine Minderheit von Pazifisten wie Bertha von Suttner, 1905 als erste mit dem Friedensnobelpreis ausgezeichnet, die noch bis kurz vor ihrem Tod im Jahr 1914 vor dem Ausbruch des Krieges gewarnt hatte, oder Gustav Landauer, dessen Freundschaft mit Martin Buber an diesem Krieg zerbrach, waren die Ausnahme.

Auch Georg Simmel entgeht der patriotischen Stimmung nicht. Seine Haltung hat er in dem Buch Der Krieg und die geistigen Entscheidungen, im Juni 1917 in München bei Duncker \& Humboldt erschienen, dokumentiert. 
Im Werbetext des Verlags, der vermutlich von Simmel selbst verfasst wurde, heißt es:

Man darf von diesem Büchlein Wichtiges und Erleuchtendes erwarten. Es begleitet in vier Abhandlungen den Krieg mit der Kraft eines visionären Durchblickes und zeigt die Wandlungen in der Beurteilung des Krieges und der Weltlage von der vollen inneren Zuversicht aus der großen Zeit der ersten Kriegswinters in dem ersten Aufsatz bis zu den zurückhaltenden Gedanken über den positiven Ertrag dieses Krieges in der letzten Abhandlung ,Die Idee Europa'. (Simmel 2017, 429).

Auch wenn Simmel den Krieg anfangs zuversichtlich sah, sollte er dennoch nicht einfach in eine Reihe mit den Euckens und Schelers gestellt werden, er schreibt mit deutlich weniger Schaum vor dem Mund, gleich zu Beginn klingen schon leise Zweifel und Zwischentöne an.

Bekanntlich haben Georg Lukács und Ernst Bloch sich wegen Simmels Haltung zum Krieg von ihm entfernt, Bloch, der so nachhaltig von Simmel beeinflusst wurde, mit ihm sogar drei Wochen Ferien in Italien verbrachte und seine Suppen genoss (vgl. Zudeick, S. 38), kündigt ihm die Freundschaft, auch wenn er sich bereits intellektuell wegen Simmels „gefräßigem“ Relativismus von ihm abzuwenden begonnen hatte. Aus Heidelberg schreibt er Simmel einen Brief:

„Sie haben niemals eine definitive Antwort auf etwas gesucht, niemals. Das Absolute war Ihnen vollkommen suspekt und verschlossen. Heil Ihnen! Nun haben Sie es endlich gefunden. Das metaphysische Absolute ist jetzt der deutsche Schützengraben.“ (Zudeick, 1985, 38)

Noch einmal kommt es zu einem zufälligen Treffen in Heidelberg, das Bloch selbst so darstellte: , Ich habe ihn nicht gegrüßt, er aber grüßte mich, und davon war ich doch ergriffen. Ich habe kühl geantwortet, aber ich ging in seinen Vortrag. Der war entsetzlich. Es war ein einziger ProKriegs-Vortrag, alldeutsch bis zum Exzeß, völlig unbegreiflich. Und das war das Ende." (Zudeick, S. 39).

Bloch hat Recht und Unrecht zugleich. „Sie haben niemals eine definitive Antwort gesucht", dies charakterisiert gewiss das Denken Simmels und liefert doch auch schon den Grund, warum Simmel nicht umstandslos in die Reihe der Kriegsenthusiasten gestellt werden sollte, dafür war sein Denken zu abwägend, eben nicht „definitiv“ genug.

Im November 1914 hält Georg Simmel eine Rede in Straßburg mit dem Titel „Deutschlands innere Wandlung“. Da war bei so manchem Frontsoldaten die Anfangsbegeisterung bereits vorbei, hatten die 
deutschen Truppen ihre Kriegsverbrechen im neutralen Belgien schon begangen. Am 23. August das Massaker von Dinant, vom 25. bis zum 28. August zerstörten sie Löwen, einen großen Teil der Häuser und auch die Universitätsbibliothek, wenige Wochen später brannte die Kathedrale von Reims.

Als Reaktion auf die Anschuldigungen dieser Kriegsverbrechen aus dem Ausland veröffentlichten 93 Wissenschaftler und Kulturschaffende das Pamphlet: „An die Kulturwelt" Ein Aufruf“, das unter anderen von Max Planck, Max Reinhardt und Gerhat Hauptmann unterzeichnet wurde und den deutschen Militarismus verteidigte. Simmel zählte nicht zu den Unterzeichnenden, aber sein Name findet sich auf der Erklärung der Hochschullehrer des Deutschen Reiches vom Oktober 1914. Über 4000 Professoren, also etwa $80 \%$ aller Dozenten an deutschen Universitäten, unterzeichneten dieses Dokument in der Nachfolge des Manifests der 93.

Von all diesem Nationalismus findet sich in Simmels Rede wenig, auch Kaiser Wilhelm oder Politiker kommen nicht vor, ebenso wenig wie konkrete Kriegsberichte. Von einem Hurrapatriotismus ist Simmel zumeist recht weit entfernt. Und die Basis bilden Gedanken, die Simmel schon in seiner Philosophie des Geldes formuliert hatte. Die Stichwörter dieser Rede sind zwar „der neue Mensch“, das „,andere Deutschland“, Volk, Seele und Schicksal, aber auch Materialismus, Mammonismus, Geldgier, Gewinn- und Genußsucht. Und wenn der Krieg eben auch "dem Leben eine „ungeheure Intensitätssteigerung gebracht“ hat, so werden zwar „die wundervollen Menschen noch wundervoller“, aber eben auch die „Lumpen noch lumpiger" (Simmel 2017, 19). Und auch die Folgen dieses Krieges bedenkt Simmel:

Deutschland wird, auch bei glücklichem Ausgang des Krieges, vergleichsweise arm zurückbleiben. Was an Industrien, an Handelsverbindungen, an Einrichtungen, an gut begründeten wie an gewagten Unternehmungen heute schon zusammengebrochen ist, was durch Stillstand der Betriebe verlorengegangen ist, kann kein Mensch übersehen. (Simmel 2017, 17)

Dennoch sieht er in dem Krieg eine Chance, sieht ihn als Katharsis, als Vollendung von 1870, die Überwindung des Mammonismus und des Transzendentwerdens des goldenen Kalbes. Krieg bezeichnet Simmel als den „großen Scheidungsprozeß zwischen Licht und Finsternis“. Wie einst Novalis' ${ }^{6}$ Heinrich von Ofterdingen die blaume Blume im inneren der Erde fand, sieht auch Simmel die Rettung in der Tiefe, der Krieg soll - gewissermaßen als natürliches Ereignis - alles Oberflächliche, die Schichten, die sich in Friedenszeiten anlagern und das Wesentliche 
so überlagern, wegsprengen und den ideellen Kern deutschen Wesens freilegen.

Auf einen weiteren Aspekt dieser Hoffnung auf Deutschland als ein geeintes Volk hat Margarete Susman ${ }^{2}$ bereits hingewiesen, die Hoffnung der Juden, endlich dazuzugehören, hatte Wilhelm II. nicht verkündet - Ich kenne keine Parteien mehr, kenne nur noch Deutsche? Von Ernst Toller, er selbst auch Jude und Kriegsfreiwilliger der ersten Stunde, wissen wir von seinem Wunsch nach Aufnahme in die nationale Gemeinschaft, die der Krieg nun angeblich bringen sollte, so wie in Kafkas Utopie einer großen Gemeinschaft, dem „Naturtheater von Oklahoma“, wo alle, wirklich alle Willkommen sind, ergreift man nur rechtzeitig die Chance.

Einen Beitrag, den Simmel nicht in den Band Der Krieg und die geistigen Erscheinungen aufnahm, veröffentlichte er unter dem Titel Europa und Amerika im Berliner Tageblatt vom 4. Juli 1915. Wegen dieses Artikels wollte der stellvertretende Gouverneur von Straßburg ein Verfahren wegen „undeutschen Verhaltens“ einleiten, das aber letztlich nicht zustande kam (vgl Simmel 2017, 429). Dieser Beitrag zeigt bereits eine gewachsene Skepsis und macht zugleich deutlich, wie sehr Simmel europäische geistige Traditionen gefährdet sieht und sie gegen die Amerikanisierung der Welt, die Mechanisierung, die Moderne bewahren will. Als Garant dafür führt er Europa ins Felde, schließlich liege ja auch Deutschland in Europa: „Europa steht im Begriff, Selbstmord zu begehen, und Amerika sieht darin die Chance für sich selbst, sich an die Spitze des Weltgeschehens zu setzen. Es steht dabei, wie der lauernde Erbe am Sterbebett des reichen Erblassers." (Simmel 1915, 2)

Und sicherlich waren die Apologeten des Kriegs und deutscher Mission nicht begeistert zu lesen, dass es ,weltgeschichtlich (...) ziemlich gleichgültig (ist), ob diese vierzehntausend Quadratkilometer von ElsassLothringen (an Umfang und Bevölkerung etwa der vierzigste Teil von Deutschland) deutsch oder französisch sind, fast so gleichgültig, wie ob das Trentino zu Österreich oder zu Italien gehört.“ (a.a.O.. 3))

Indem Simmel Amerika als den eigentlichen Gegner ausmacht, gelingt es ihm, Europa wieder zu vereinen. Wenn auch nur auf dem Papier. Denn:

2 Vgl. Susmann, Margarete (1959, 24): "Und daß dies besonders bei den deutschen Juden der Fall war, daß sie patriotischer waren als die große Mehrzahl der Deutschen, ist nur auf den ersten Blick befremdlich, denn dieser Patriotismus war darum so echt, weil Deutschland für sie nicht nur die Heimat, sondern zugleich die Fremde war." 
„Schließlich wohnt Europa in einem Hause und Amerika in einem anderen. Und der Frevel nicht nur, sondern die Kurzsichtigkeit und der Wahnwitz unserer Gegner, die diesen Krieg entzündet haben, ist wie von Wohnparteien, die eine andere Partei, weil sie allerhand gegen sie auf dem Herzen haben, aus ihrer Wohnung vertreiben wollen und darum das ganze Haus anzünden, ihr eigenes Haus.“ (a.a.O., 4 f.)

Diese Haltung und seine Sorge vor dem „Selbstmord Europas“, wie er es auch in einem Brief an Herman Keyserling nannte, werden Simmels Haltung zum Kriege bis zu seinem Lebensende bestimmen, auch in Die Idee Europa schreibt er gegen die Internationalisierung, heute würden wir wohl Globalisierung sagen. Letztlich waren die Zweifel wohl doch stärker als die Zuversicht in deutsche Größe oder, wie Margarete Susmann es nannte: die „absolute Situation“, das größte Ereignis seines Lebens.

Zweifel ist ein Wort, das wir in Zusammenhang mit Stefan George und seiner Person eher selten vernehmen. 1901 veröffentlicht Georg Simmel: Stefan George. Eine kunstphilosophische Studie. Bis heute eine der interessantesten Arbeiten zur Lyrik Georges. Hellsichtig beschreibt er darin als erster das ästhetische Projekt Georges:

Seine Kunst wird von vornherein durch das Bestreben bezeichnet, ausschließlich als Kunst zu wirken. Während sonst die Endabsicht des Lyrikers in dem Gefühls- oder Vorstellungsinhalt zu liegen pflegt, zu dessen Darstellung und Erregung ihm die künstlerische Form als Mittel dient, - ist hier die grundsätzliche Wendung vollzogen: daß umgekehrt aller Inhalt das bloße Mittel ist, um rein ästhetische Werthe zu bilden. (Simmel 1901, 208)

Dieses Verhältnis von Inhalt und Form, das rein Ästhetische als absoluter Wert ist das kennzeichnende Prinzip im Werk und auch im Leben Georges.

Simmel, der „für alle geistigen Feinschmecker“ als „die wichtigste Sehenswürdigkeit des Berlin um 1900“ galt (Karlauf, 491) und dessen Bekanntschaft mit George im Oktober 1897 stattfand, war dann auch mitentscheidend für den literarischen Durchbruch Georges. Stefan George selbst bewunderte Simmels Intellektualität, aber gestand auch, das er nicht allzu viel von seinen Überlegungen verstehe. „Man kann ganz vollkommen sein“, fasste er 1920 sein Verhältnis zu Simmel pointiert zusammen, „und von alledem nicht das Geringste verstehen." (Karlauf, 523)

Manches ließe sich hier noch zu diesem Verhältnis anführen, dass Simmel "das Biographische dem Werk gegenüber immer als unwichtig erschienen (ist)" wie Margarete Susmann $(1959,1)$ feststellte, teilte er mit George; oder etwa die Bedeutung der Begegnung mit George für den Gedanken eines individuellen Gesetzes, wie Klaus Christian Köhnke 
schrieb. (nach Karlauf, S. 521) oder auch das Verhältnis Georges zu Gertrud Simmel und Gertrud Kantorowicz. Hier aber soll es jetzt um George und den Ersten Weltkrieg gehen.

Im Juli 1917 veröffentlich Stefan George im Verlag Georg Bondi und als Sonderdruck das Gedicht „Der Krieg“. Die acht Seiten erscheinen mit einer Auflage von 6500 Exemplaren, für George eine außerordentlich hohe Auflage, die die Bedeutung dieses Gedichts unterstreicht.

Es besteht aus zwölfStrophen mit jeweils zwölf Versen, die als jambische Fünfheber mit gelegentlichen daktylischen Einsprengseln eine Länge von zehn bis zwölf Silben erreichen. Dieses Volumen bietet Raum für eine gründliche Erörterung des Themas. Das jambische Gleichmaß, das nur bei weiblichen Kadenzen und daktylischen Einfügungen etwas belebt wird, verleiht dem Gedicht einen festen Ton, der das inhaltliche Pathos stützt. Reimlosigkeit gibt dem Gedicht den Charakter einer Rede, die den inhaltlichen Ernst nicht durch poetischen Schmuck überspielen will. Die Zahl der Strophen lässt an das neue Jerusalem denken, das - laut Geheimer Offenbarung - zwölf Tore mit zwölf Engeln darauf und zwölf Grundsteine mit den Namen der Apostel hat. (Helmuth Kiesel, FAZ, S. 2)

George stilisiert sich in diesem Gedicht zum Seher und besteht auf seiner Außenseiterposition: „Am streit wie ihr ihn fühlt nehm ich nicht teil" (George 1928, 29). George gefiel sich bekanntlich zeitlebens in der Position des charismatischen Führers oder gar als neuer Jesus, der seine Apostel um sich schart. Aber auch wenn er sich vom Kriegsgeschehen distanziert, ist seine Haltung zumindest ambivalent. In dem George-Buch seines Hofbiographen Friedrich Wolters, 1930 unter intensiver Mitarbeit Georges erschienen, ließ er sich auch schon zu Kriegsbeginn als Seher darstellen:

Als der Krieg ausbrach, weilte George mit einigen älteren und jüngeren Gefährten in der Einsamkeit der Berner Hochalpen. Keiner von denen die ihn damals sahen, wird das tiefe Leid vergessen, das ihn überfiel und den Niegebeugten fast niederdrückte mit seiner Last. Mit einem Blicke sah er das ungeheuere Geschehen aufgerollt: die ganze Wucht dessen was er oft geahnt und die ganze Furchtbarkeit dessen was nun kam, stürzte mit einem Male auf ihn ein: Das Schicksal, das er längst vorausgesagt hatte, auf sein Volk und damit auf die Seinen. (Wolters 1930, 437)

Thomas Karlauf indes stellt die Geschichte in seiner GeorgeBiographie prosaischer dar. Besorgt war George erst einmal um sein eigenes Schicksal. Bei Kriegsausbruch verbrachte er seine Ferien in der Schweiz. Auf dem deutschen Konsulat in Bern erfuhr er, dass er, da 
er soeben 46 Jahre alt geworden war, auch nicht mehr zum Landsturm oder zur Reserve gehörte. „Wegen der erheblichen Einschränkungen im Zugverkehr empfahl man ihm auf dem Konsulat, vorerst nicht zu reisen, und so fuhr George ins Berner Oberland zurück, wo er seine Ferien bis Ende August fortsetzte.“ (Karlauf, S. 1027)

Wie unerwartet diese Haltung selbst für die engsten Freunde war, zeigt auch die Reaktion Friedrich Gundolfs, der unbedingt mit einer aktiven Kriegsteilnahme Georges gerechnet hatte. Schließlich teilten beide die Diagnose der Moderne, der entzauberten Welt, wie Max Weber es nannte. Bei Gundolf klingt die Beschreibung der Vorkriegszeit so:

Wir gingen einer entstellten und erkalteten menschheit entgegen die sich mit ihren vielspältigen errungenschaften und verästelten empfindungen brüstete, indessen die große tat und die große liebe am entschwinden war. Massen schufen gebot und regel (...) und dreistes lachen verkündete den Untergang des heiligtums. (Gundolf, 214)

George aber teilte Gundolf brieflich mit: „Nichts wird so heiss gegessen als es gekocht wird““ (...) ,Ich sehe keinen grund vorläufig in eile die schweiz zu verlassen. “" (Karlauf 2007, 1027)

Dennoch sah George wie Simmel im Krieg auch die Möglichkeit einer großen Reinigung. Im Stern des Bundes heißt es schon 1914:

Zehntausend muß der heilige wahnsinn schlagen

Zehntausend muß die heilige seuche raffen

Zehntausende der heilige krieg.

(George 1922, 25)

Auch viele andere Aussagen Georges zeigen, wie ambivalent seine Haltung war, überliefert ist von ihm im Ersten Weltkrieg der Satz: „Nur wenn die gelben Affen kommen, dann nehme ich selbst die Flinte“. (vgl. Mattenklott 1985, 215). Eine Haltung, die er mit Thomas Mann teilte, der sich in seinen Gedanken im Kriege echauffierte: „Man glaubt, ein Recht zu haben, auf Deutschland Kirgisen, Japaner, Gurkas und Hottentotten loszulassen, - eine Beleidigung, beispiellos, ungeheuerlich..." (Mann 1976, 36). Humanistische Überlegungen haben George sicher nicht von der Teilnahme am Krieg abgehalten.

Von den Mitgliedern des Kreises forderte George jedoch wie immer Selbstverleugnung und Härte, das betraf auch die Teilnahme am Krieg. Im Juli 1918 töteten sich Bernhard von Uxkull und Adalbert Cohrs, nachdem sie bei dem Versuch, in die Niederlande zu desertieren, verraten und verhaftet worden waren. Bei einem letzten Besuch im Harz hatte George 
Unverständnis gezeigt, dass die vom Krieg schwer traumatisierten Freunde nicht mehr an die Front zurück wollten. George antwortet Cohrs mit einem Vierzeiler:

Du hast des lebens götterteil genossen

Von glück und rausch und schwärmen wunderbar..

Du darfst nicht murren, ward dir nun beschlossen

Des wahren lebens ander teil: gefahr. (in: Karlauf 2007, 1063)

George, dieser selbst ernannte Führer der Jugend, sah Politik und Kunst, die Welt des Geistigen immer als getrennte Welten. In seinem Staat, dem „geheimen Deutschland“, dem der Kunst und der Erziehung von kleinen Eliten, finden sich Ansichten, Haltungen, die dem deutschen Militarismus gleichen. Aber der Politik will George keine Macht über sein Reich einräumen. Die Verweigerung des Kriegs lässt sich so auch als eine Staatskrise verstehen. Der Krieg war für George ein Parallelereignis, in dem sich die Teilnehmer auch bewähren konnten - nicht aber er selbst. Seine Idee der Kunst, der Welt des Ästhetischen war absolut, der Krieg als reales Ereignis zweitrangig, vor allem Material für ästhetische Aneignung, mehr nicht. Im Gegensatz zu Simmel lassen seine Aussagen keine Zweifel erkennen, schließlich war er Seher, kein Philosoph oder Soziologe.

\section{Bibliographie}

Clark, Christopher (2013), The Sleepwalkers. How Europe went to war in 1914. London: Penguin Books.

George, Stefan (1922), Der Stern des Bundes. Berlin: Georg Bondi.

George, Stefan (1928). Das neue Reich. Berlin: Georg Bondi.

Gundolf, Friedrich (1920), George. Berlin: Georg Bondi.

Karlauf, Thomas (2007), Stefan George. Die Entdeckung des Charisma. München: Blessing Verlag ebooks.

Helmuth Kiesel (2017), „Stefan Georges Kriegslyrik: Hochpriesterlichseherische Verkündigung“. https://www.faz.net/aktuell/feuilleton/ buecher/stefan-georges-kriegslyrik-hochpriesterlich-seherischeverkuendigung-15126540.html (Stand: 10.01.2018)

Mann, Thomas (1914), „Gedanken im Kriege“. In: ders. (1977) Essays, Band 2. Politik. Hrsg. v. Hermann Kurzke. Frankfurt am Main: Fischer Verlag, 23 -37 .

Mattenklott, Gert (1985), Bilderdienst. Ästhetische Opposition bei Beardsley und George. Frankfurt am Main: Syndikat Verlag.

Natorp, Paul (1918), Deutscher Weltberuf. Geschichtsphilosophische Richtlinien. Jena: Eugen Diederichs. 
Nietzsche, Friedrich (1980), „Unzeitgemäße Betrachtungen“. In: ders.: Werke in sechs Bänden, hrsg. Von Karl Schlechta, München: Hanser Verlag.

Simmel, Georg (1901), „Stefan George. Eine kunstphilosophische Studie“. In: Neue Deutsche Rundschau (Freie Bühne) 12, 2 (1901), S. 207-215.

Simmel, Georg (2017), „Der Krieg und die geistigen Entscheidungen“. In: ders. Der Krieg und die geistigen Entscheidungen. Gesamtausgabe Band 16. Hrsgg. v. Fitzi, G; Rammstedt O., Frankfurt am Main: Suhrkamp Verlag.

Simmel, Georg (1915), „Europa und Amerika - Eine weltgeschichtliche Betrachtung“, ex: Berliner Tageblatt, 44. Jg., Nr. 336, 4. Juli 1915, Morgen-Ausgabe, 2. Beiblatt. http://socio.ch/sim/verschiedenes/1915/ europa amerika.htm (Stand: 03.01.2018)

Susmann, Margarete (1959), Die geistige Gestalt Georg Simmels. http://www. margaretesusman.com/geistigegestaltsimmel.htm (Stand: 06.02.2018)

Wolters, Friedrich (1930), Stefan George und die Blaetter fuer die Kunst : dt. Geistesgeschichte seit 1890. Berlin: Georg Bondi.

Zudeick, Peter (1985), Der Hintern des Teufels. Ernst Bloch - Leben und Werk. Moos \& Baden-Baden: Elster Verlag.

\section{RESUMO \\ Georg Simmel, Stefan George e a Grande Guerra}

Inicialmente, a Grande Guerra foi recebida com euforia por muitos escritores e filósofos, entre eles Max Weber, Martin Buber, Max Scheler e também Georg Simmel. Para Simmel, a guerra era sinónimo de uma maior intensidade da vida, uma maneira de enfrentar / superar os nivelamentos da sociedade causados pela adoração do dinheiro ("Mammonismus"). Esta exaltação dos intelectuais perante a guerra não era comum a todos - conhecidas são as críticas duras de Georg Lucácz e Ernst Bloch ao entusiasmo bélico de Simmel.

Sobre Stefan George, Simmel escreveu em 1901: „A sua arte é conhecida desde o início pelo desejo de agir exclusivamente como uma arte (...) a mudança fundamental é completa: que, ao contrário, todo o conteúdo é o mero meio para formar valores puramente estéticos.“ Assim, Simmel reconhece na estética de Stefan George a razão a partir da qual George vai rejeitar a guerra - ao contrário de muitos membros do círculo de George (George-Kreis) - e que tem a sua expressão no poema "A Guerra", publicado pela primeira vez em 1917.

Este contributo pretende demonstrar as razões filosóficas e estéticas relativamente ao entusiasmo pela guerra e a sua rejeição por Simmel e George, atitudes que não podem ser explicadas pela oposição militarismo/pacifismo, normalmente utilizada para distinguir apoiantes e críticos da guerra.

Palavras-chave: Georg Simmel - Stefan George - Grande Guerra - militarismo - pacifismo 


\section{ABSTRACT \\ Georg Simmel, Stefan George and the Great War}

Initially, the Great War was received euphorically by many writers and philosophers, including Max Weber, Martin Buber and Max Scheler along with Georg Simmel. For Simmel, the war was synonimous with a greater pace of life, a form of dealing with / overcoming the levelling of society caused by the worshipping of money ('Mammonismus'). This exaltation of the war on the part of intellectuals was not common to all - the harsh criticisms of Simmel's enthusiasm for the war on the part of Georg Lucácz and Ernst Bloch are well-known.

Regarding Stefan George, in 1901 Simmel had written: 'His art has been known since its beginning for the wish to act exclusively like an

art (...) the fundamental change is complete: that on the contrary, all content is merely the means for forming values that are purely aesthetics.' Therefore, in the aesthetic of Stefan George, Simmel acknowledges the reason that George will reject the war - contrary to many members of George's circle (George-Kreis) and has its expression in the poem 'The War', first published in 1917.

This contribution seeks to demonstrate the philosophical and aesthetical reasons for enthusiasm for the war and its rejection by Simmel and George, attitudes that are not able to be explained by the opposition to militarism/pacifism that is normally deployed to distinguish between supporters and critics of the war.

Keywords: Georg Simmel - Stefan George - Great War - - militarism - pacifism 